\title{
On the Reflected Appraisals Dynamics of Influence Networks with Stubborn Agents
}

\author{
Anahita Mirtabatabaei, Peng Jia, Noah E. Friedkin, Francesco Bullo
}

\begin{abstract}
This article focuses on the evolution of interpersonal influences in a group of stubborn individuals as they discuss a sequence of issues. Each individual opinion about a single issue is updated based upon the convex combination of the individual's current opinion, the neighbors' current opinion, and the individual's initial opinion; the attachment to the initial opinion characterizes how stubborn an individual is. To model the evolution of the influence network, we employ Friedkin's "reflected appraisal" model: each individual's selfweight on a new issue is determined by the individual's average influence and relative control on other individuals on prior issue outcomes. These modeling assumptions lead to a dynamical system for the evolution of self-weights. We establish the well-posedness and continuity of the proposed dynamics and prove the existence and uniqueness of equilibria for stubborn individuals. We then study the impact of network topology on the individuals' final self-weights. We prove the convergence of all system trajectories for the special case of doubly-stochastic networks and homogeneous stubbornness. We characterize equilibrium self-weights for systems with centralized networks and heterogeneous stubbornness. Finally, our numerical simulations illustrate how existence, uniqueness and attractivity of the equilibria holds true for general network topologies and stubbornness values.
\end{abstract}

\section{INTRODUCTION}

In many real world complex systems ranging from biological to social networks, one crucial aspect is discovering the interrelationship between the evolution of network topology and the dynamics of nodes, so called co-evolutionary or adaptive networks. Much literature has focused on adaptive networks whose evolution is based on the idea that "similarity breeds connection" [17], that is, interactions are formed in view of the similarity of nodes' dynamics. Accordingly, in models of opinion dynamics, neighboring relation is defined based on bounded confidence [13], [4], [2] or influence [18], which means that an individual only interacts with those whose opinions are close enough to her own.

Recently, there has been a growing interest in the study of influence networks and opinion formation in the field of social psychology [7], [12], [5], [9]. A big challenge in this field has been the investigation of influence change based on social comparison. Following this trend, here we describe the co-evolution of social influence networks and opinion

This work was supported by the UCSB Institute for Collaborative Biotechnology through grant W911NF-09-D-0001 from the U.S. Army Research Office. The content of the information does not necessarily reflect the position or the policy of the Government, and no official endorsement should be inferred.

The authors are affiliated with the Department of Mechanical Engineering and the Center for Control, Dynamical Systems and Computation, University of California at Santa Barbara, \{mirtabatabaei,pjia, bullo\}eengineering.ucsb.edu.

Noah Friedkin is affiliated with the Department of Sociology, University of California at Santa Barbara, friedkin@soc.ucsb.edu. formation in a group of individuals discussing a series of issues via a reflected appraisal mechanism [8], [3], [11]. This mechanism is based on the psychological axiom that "our self-concepts are formed as reflections of the responses and evaluations of others in our environment" [22], and is supported by empirical investigations [22], [8]. In this context, (Friedkin, 2011) developed a model of influence network evolution whose dynamics is described in two stages. First, with regards to single-topics decision making, each individual updates her opinion through a convex combination of the individual's current opinion, the neighbors' current opinion, and the individual's initial opinion [9]. Second, with regards to multi-topics decision making, the update mechanism of reflected appraisal is adopted. More specifically, this mechanism asserts that each individual's self-weight on a new issue is determined by her average influence and relative control on other individuals on prior issue outcomes [8]. As an individual's self-weight varies, the relative weights that she assigns to other individuals and thus the relative influence network changes. A variation of Friedkin's model has been analyzed in [15], [14], where with regards to single-topics decision making, the DeGroot's first-order model of opinion dynamics is considered [5]. This model "predicts that the social power ranking among individuals is asymptotically equal to their centrality ranking" [15] and characterizes critical social network properties leading to the emergence of single opinion leaders, clusters of leaders, or diffuse and democratic power structures. Another alternative form of Friedkin's model has been explored where opinion evolution is based on randomized gossip typologies [6].

In this article, we study another variation of Friedkin's model of influence network evolution on a group of stubborn individuals, which comes closer to the original formulation. In their work, (Friedkin and Johnsen, 1999) assumed that each individual adheres to her initial opinion to a certain degree and this stubbornness is equal to her self-weight, that is, her social power, and thus topic-dependent. We investigate stubbornness factors that are agent-dependent, independent of issues and individuals' self-weights. We investigate how this stubbornness of exogenous opinions in single-topic opinion dynamics effects the evolution of relative power over a series of topics. In the literature [1], [19], stubborn individuals have been recognized to play a role in shaping opinion profiles by preventing consensus and leading to limit behaviors which are more complex than an agreement condition. In these studies, stubborn individuals typically do not change their opinions despite social interactions. However, we assume that all individuals are to some degree stubborn against new ideas. 
The contributions of this paper are five-fold. First, we introduce a variation of Friedkin-Johnsen model where we adopt real continuous variables to represent heterogeneous agent-dependent stubbornness. We characterize the technical conditions under which the model is well-posed. Second, we demonstrate the continuity of the proposed dynamics and the existence and uniqueness of equilibira for comparatively stubborn individuals. Third, we consider such a model under the condition of homogeneous stubbornness and doubly stochastic relative interaction network. We establish that in the corresponding dynamical system: (i) the unique equilibrium self-weights vector has uniform entries, a notion of democracy, that is defined to be a situation where agents have equal rights; and (ii) the system exponentially converges to this equilibrium vector. Forth, we study the model with heterogeneous stubbornness and centralized relative interaction network. We establish that (i) the equilibrium self-weights vector is unique if the central individual is stubborn enough; (ii) for homogeneous stubbornness, the center individual reaches highest self-weight, however, for heterogeneous stubbornness, it is possible for a non-central individual to obtain the highest self-weight. Finally, our numerical simulations illustrate how existence, uniqueness and attractivity of the equilibria, that are independent of initial conditions, holds true for general network topologies and stubbornness values.

Notation. In the Euclidean space $\mathbb{R}^{n}$, we define $\mathbb{1}_{n}=$ $(1, \ldots, 1)^{T}$ and $\mathbb{O}_{n}=(0, \ldots, 0)^{T}$. Moreover, for $i \in$ $\{1, \ldots, n\}$, we let $\mathbb{e}_{i}$ be the vector with all entries equal to 0 except for the $i$ th entry equal to 1 . Given $\left(x_{1}, \ldots, x_{n}\right) \in \mathbb{R}^{n}$, let $\operatorname{diag}(x)$ denote the diagonal $n \times n$ matrix whose diagonal entries are $x_{1}, \ldots, x_{n}$. We also recall the definition of the $n$-simplex $\Delta_{n}=\left\{x \in \mathbb{R}^{n} \mid x \geq 0, \mathbb{1}_{n}^{T} x=1\right\}$.

\section{THE MATHEMATICAL MODEL}

In the mathematical modeling of the opinion evolution in a group of decision makers discussing a sequence of issues, two separate dynamics are considered: (i) opinion evolution on one issue while the influence network is fixed; and (ii) the evolution of influence network based on individuals' reflected appraisals. We consider a group of $n \geq 2$ individuals discussing a sequence of issues $s \in \mathbb{N}$. We aim to discover the dynamics of individuals' relative measures of self-weight $x(s) \in \Delta_{n}$ as the issue under discussion changes. Specifically, the model is defined as follows.

\section{A. Opinion dynamics on single issue}

Discussing any issue $s \in \mathbb{N}$, as time $t \in \mathbb{R}_{\geq 0}$ progresses, each individual $i \in\{1, \ldots, n\}$ updates her opinion $y_{i}(s, t) \in$ $\mathbb{R}$ about that issue by considering her initial opinion, her current opinion, and the current opinions of peers.

$$
\begin{aligned}
y_{i}(t+1, s)=\left(1-\theta_{i}\right) y_{i}(0, s) & +\theta_{i} x_{i}(s) y_{i}(t, s) \\
& +\theta_{i}\left(1-x_{i}(s)\right) \sum_{j=1}^{n} c_{i j} y_{j}(t, s),
\end{aligned}
$$

where $\theta_{i} \in[0,1]$ denotes $i$ 's susceptibility to influence by peers (i.e., $1-\theta_{i}$ represents $i$ 's stubbornness) and the coefficients $c_{i j}$ are the fixed relative interpersonal weights that $i$ accords to other individuals, that is, the $n \times n$ matrix $C$ is the relative interaction matrix that is row-stochastic with zero diagonal [9]. Note that self-weights $x_{i}(s) \in[0,1]$ are fixed over each topic $s$, while $\Theta=\operatorname{diag}(\theta)$ and $C$ are independent of topic and time. Then, the vector of opinions about any issue $s$ is a trajectory $t \mapsto y(s, t) \in \mathbb{R}^{n}$ that evolves as follows:

$$
y(t+1, s)=\Theta W(s) y(t, s)+\left(I_{n}-\Theta\right) y(0, s),
$$

where, following [8], the $n \times n$ weight matrix $W(s)$ is rowstochastic with diagonal entries $w_{i i}(s)=x_{i}(s)$,

$$
W(s)=\operatorname{diag}(x(s))+\left(I_{n}-\operatorname{diag}(x(s))\right) C .
$$

The dynamical system (1) implies that each individual $i$ updates her opinion considering her initial opinion scaled by her stubbornness $1-\theta_{i}$ and the current opinion of herself and peers scaled by her susceptibility $\theta_{i}$. Moreover, $i$ scales her own current opinion by her self-weight $x_{i}(s)$ and peers' current opinions by her lack of self-confidence $1-x_{i}(s)$.

Before proceeding, we introduce an assumption that is employed in the description of self-weights dynamics across issues. First, let us quote a few relevant definitions from graph theory. A node of a digraph with out-degree zero is named a sink. A maximal subgraph of a digraph that is strongly connected forms a strongly connected component $(S C C)$. For any digraph $G$, if each of its strongly connected components is contracted to a single vertex, then the result is an acyclic digraph that is condensation digraph of $G$. Therefore, any condensation digraph contains at least one sink. We define sink components of $G$ to be the sinks of $G$ 's condensation directed graph. Moreover, we denote the digraph associated with the weights matrix $W(s)$ by $G(W(s))$, that is, $W(s)$ is the adjacency matrix of $G(W(s))$.

Assumption II.1 (Stubborn sinks). Any sink component of $G(W(1))$ contains at least one node $i$ such that $\theta_{i}<1$.

Intuitively speaking, this assumption implies that a group of individuals who do not let other groups influence their opinions (i.e., a sink component of weights graph) can not be highly flexible toward new ideas (i.e., all $\theta_{i}$ 's equal to one). Assumption II.1 also implies that $\Theta \neq I_{n}$. If we adopt $\Theta=$ $I_{n}$ for the introduced dynamical system in this paper, then the resulting system is equivalent to the so called DeGrootFriedkin system and is studied in [14].

\section{B. Dynamics of self-weights across a sequence of issues}

For the evolution of individuals' self-weights as they discuss a sequence of issues, we consider the reflected appraisal mechanism, where each individual's self-weight on one issue is set equal to the relative power that the individual exerted over the prior issue outcome [8].

The reflected appraisal mechanism updates the selfweights vector via $x(s+1)=V(s)^{T} \mathbb{1}_{n} / n$, where $V(s)$, if it exists, is determined by $\lim _{t \rightarrow \infty} y(s, t)=V(s) y(s, 0)$. The dynamical system (1) for any $s \in \mathbb{N}$ and all $t \geq 1$ is 
equivalent to

$$
\begin{aligned}
& {\left[\begin{array}{c}
y(s, t) \\
y(s, 0)
\end{array}\right]=\left[\begin{array}{cc}
\Theta W(s) & I_{n}-\Theta \\
0 & I_{n}
\end{array}\right]\left[\begin{array}{c}
y(s, t-1) \\
y(s, 0)
\end{array}\right]} \\
& =\left[\begin{array}{cc}
(\Theta W(s))^{t} & \sum_{\tau=0}^{t}(\Theta W(s))^{\tau}\left(I_{n}-\Theta\right) \\
0 & I_{n}
\end{array}\right]\left[\begin{array}{l}
y(s, 0) \\
y(s, 0)
\end{array}\right]
\end{aligned}
$$

Hence, individuals' eventual influence on each other equals

$$
V(s)=\lim _{t \rightarrow \infty}\left((\Theta W(s))^{t}+\sum_{\tau=0}^{t}(\Theta W(s))^{\tau}\left(I_{n}-\Theta\right)\right) .
$$

In Lemma III.1, we show that under Assumption II.1, $\Theta W(s)$ is strictly row-substochastic for all $s \in \mathbb{N}$. A square matrix $A$ is strictly row-substochastic, if its entries are nonnegative, every row adds up to at most one, and for any SCC of $G(A)$, its associated adjacency matrix contains at least one row whose sum is strictly less than one. The spectral radius of a strictly row-substochastic matrix is strictly less than one. Then, $\lim _{t \rightarrow \infty}(\Theta W(s))^{t}=0_{n}$ and, according to [10, Section 5.6], $\lim _{t \rightarrow \infty} \sum_{\tau=0}^{t}(\Theta W(s))^{\tau}=\left(I_{n}-\Theta W(s)\right)^{-1}$. Thus, under Assumption II.1, $V(s)$ exists and is equal to

$$
V(s)=\left(I_{n}-\Theta W(s)\right)^{-1}\left(I_{n}-\Theta\right) .
$$

We refer to the set of dynamical system (3) together with $x(s+1)=V(s)^{T} \mathbb{1}_{n} / n$, that determine self-weights evolution over a sequence of issues with individuals' constant susceptibility to exogenous opinions, as the constant FriedkinJohnsen ( $c F J)$ system.

\section{Dynamical Properties of The System}

In this section, we first determine sufficient conditions that validate the dynamics of the self-weight vector in $\mathrm{cFJ}$ systems. Next, we present an equivalent expression for the evolution of self-weights, which is employed in the analysis of system equilibria. Finally, we demonstrate existence and uniqueness of system equilibria given bounded susceptibilities for a general relative interaction network.

Lemma III.1. If a cFJ system for the first issue satisfies Assumption II.1, then, for all $s \in \mathbb{N}, \Theta W(s)$ is strictly rowsubstochastic.

Proof. First, we show that for any matrix $W(s)$ such that each sink component of $G(W(s))$ contains at least one node $i \in\{1, \ldots, n\}$ with $\theta_{i}<1, \Theta W(s)$ is strictly rowsubstochastic. The digraphs $G(\Theta W(s))$ and $G(W(s))$ have the same topology unless $\theta_{i}=0$ for some $i \in\{1, \ldots, n\}$, then the $i$ th row of $\Theta W(s)$ will be equal to zero. Hence, the SCC that contains the $i$ th node in $G(W(s))$ decomposes into a sink with zero out-degree and an SCC that assigns weight to this sink in $G(\Theta W(s))$. Therefore, the adjacency matrix associated with each sink component of $G(\Theta W(s))$ is either equal to zero or contains at least one line whose row-sum is strictly less than one. The adjacency matrices associated with the SCCs of $G(\Theta W(s))$ that are not sink components are strictly row-substochastic owing to the weight these components assign to sink components. Hence, the adjacency matrices associated with all the SCCs of $G(\Theta W(s))$ are strictly row-substochastic and hence $\Theta W(s)$ is strictly row-substochastic, which guarantees the existence of $\left(I_{n}-\Theta W(s)\right)^{-1}$.

Second, we show that under Assumption II.1, each sink component of $G(W(s))$ contains at least one node $i \in$ $\{1, \ldots, n\}$ with $\theta_{i}<1$ for all $s \in \mathbb{N}$. For any $\left.x(s) \in \Delta_{n}\right\rangle$ $\left\{\mathbb{e}_{1}, \ldots, \mathbb{e}_{n}\right\}$, equation (2) implies that the digraphs $G(C)$ and $G(W(s))$, excluding self-loops, have equal topology, and thus the same set of SCCs. However, if $x(s)=\mathbb{e}_{i}$ for any $i \in\{1, \ldots, n\}$, then the SCC of $G(C)$ that contains node $i$ decomposes into a sink node $i$ and an SCC that assigns weight to this sink in $G(W(s))$. Thus, the set of $G(C)$ 's SCCs is a subset of $G(W(s))$ 's SCCs, and hence each sink component of $G(C)$ contains at least one node $i \in\{1, \ldots, n\}$ with $\theta_{i}<1$. Moreover, from $x(s+1)=$ $V(s)^{T} \mathbb{1}_{n} / n$, for all $s>1$ and any $j \in\{1, \ldots, n\}$ such that $\theta_{j}=1, x_{j}(s)=0$. Therefore, if $x(s)=\mathbb{e}_{i}$ for any $i$ and any $s>1$, then $\theta_{i}<1$, which establishes our claim.

As discussed in the proof of Lemma III.1, if $\theta_{i}=1$ for any $i \in\{1, \ldots, n\}$, then $x_{i}(s)=0$ for all $s>1$. In other words, in the presence of partially stubborn individuals, those with full susceptibility toward new ideas $\left(\theta_{i}=1\right)$ lose their self-confidence. Consequently, for the simplicity of analysis from here on, we assume that $\theta_{i}<1$ for all $i \in\{1, \ldots, n\}$.

Theorem III.2 (Evolution of cFJ system). For $n \geq 2$, if $\theta_{i}<1$ for all $i \in\{1, \ldots, n\}$, then for all $s \in \mathbb{N}$,

(i) $x_{i}(s+1)>\left(1-\theta_{i}\right) / n$; and

(ii) the cFJ system is equivalent to $x(s+1)=F(x(s))$, where the continuous map $F: \Delta_{n} \rightarrow \Delta_{n}$ is the dominant positive left eigenvector of

$$
U(x)=\frac{\mathbb{1}_{n} \mathbb{1}_{n}^{T}}{n}-\left(I_{n}-\Theta\right)^{-1} \Theta\left(I_{n}-X\right)\left(I_{n}-C\right) .
$$

Proof. Throughout this paper, we drop the $s$ argument and denote $\operatorname{diag}(x(s))$ by $X$ in the proofs for brevity. Since $\theta_{i}<1$ for all $i \in\{1, \ldots, n\},\left(I_{n}-\Theta\right)^{-1}$ exists and Assumption II.1 holds. Hence, according to Lemma III.1, $\Theta W(s)$ is strictly row-substochastic for all $s \in \mathbb{N}$. Consequently, $V(s)$ exists and is defined by equation (3),

$$
V(s)=\left(I_{n}-\Theta X-\Theta\left(I_{n}-X\right) C\right)^{-1}\left(I_{n}-\Theta\right) .
$$

According to the $\mathrm{cFJ}$ system,

$$
x(s+1)^{T}\left(I_{n}-\Theta\right)^{-1}\left(I_{n}-\Theta X-\Theta\left(I_{n}-X\right) C\right)=\frac{\mathbb{1}_{n}^{T}}{n} .
$$

Regarding statement (i), equation (5) results in

$$
\begin{aligned}
x(s+1)^{T}\left(I_{n}-\Theta\right)^{-1}-\frac{\mathbb{1}_{n}^{T}}{n} & \\
= & x(s+1)^{T}\left(I_{n}-\Theta\right)^{-1}\left(\Theta X+\Theta\left(I_{n}-X\right) C\right) .
\end{aligned}
$$

If statement (ii) holds, then $x(s+1) \in$ interior $\Delta_{n}$, and hence all entries in the right hand side of above equation are strictly positive, which proves our claim. Next we show that statement (ii) holds independent of statement (i), and thus both statements hold true.

Regarding statement (ii), equation (5) results in $x(s+1)^{T}\left(I_{n}+\left(I_{n}-\Theta\right)^{-1} \Theta\left(I_{n}-X\right)\left(I_{n}-C\right)\right)=\frac{\mathbb{1}_{n}^{T}}{n}$. 
Since $x(s+1)$ belongs to the $n$-simplex, $x(s+1)^{T} \mathbb{1}_{n} \mathbb{1}_{n}^{T}=$ $\mathbb{1}_{n}^{T}$. Therefore, above equation leads to $x(s+1)^{T}=x(s+$ $1)^{T} U(x(s))$, where $U(x(s))$ is defined by equation (4). Now, we show that $U(x(s))$ has one simple eigenvalue equal to one whose corresponding left eigenvector $u(x(s))$ has strictly positive entries. According to the Gershgorin circle theorem, all eigenvalues of $U(x(s))$ are inside the disks centered at $1 / n-\theta_{i}\left(1-x_{i}\right) /\left(1-\theta_{i}\right)$ with radius $(n-1) / n+\theta_{i}\left(1-x_{i}\right) /\left(1-\theta_{i}\right)$ for some $i \in\{1, \ldots, n\}$. As all these Gershgorin discs lie on the left side of the line $\operatorname{Re}(\lambda)=1$ in the complex plane, the real parts of all eigenvalues of $U(x(s))$ are then less than or equal to one for any $i \in\{1, \ldots, n\}$. Since $U(x(s))$ 's row sum is equal to one, it has at least one eigenvalue equal to 1 . Furthermore, matrix $U(x(s))$ is a Metzler matrix, in which all the off-diagonal components are non-negative, in our case these entries are strictly positive. Therefore, there exists $\tau>0$ such that all entries of $U(x(s))+\tau I_{n}$ are strictly positive, and hence, according to the Perron-Frobenius theorem, the spectral radius of $U(x(s))+\tau I_{n}$, denoted by $\rho\left(U(x(s))+\tau I_{n}\right)$, is a positive real simple eigenvalue. Moreover, there exists a left eigenvector associated with $\rho\left(U(x(s))+\tau I_{n}\right)$ whose entries are strictly positive. Consequently, $U(x(s))$ has a real simple eigenvalue equal to $\rho\left(U(x(s))+\tau I_{n}\right)-\tau$, which according to the previous discussion is equal to 1 , with a unique left eigenvector $u(x(s))$ that belongs to the interior of $\Delta_{n}$.

Remark 1. Theorem III.2 states that if $\theta_{i}<1$ for all $i \in\{1, \ldots, n\}$, then for any issue $s>1$ all individuals' self-weights are strictly positive independent of the topology of $G(W(s))$. For an irreducible $W(s)$, an SCC that is not a sink component in $G(W(s))$ have no influence on the final opinions of the sink components. However, the eventual influence of non-sink SCCs on the issue outcome is non-zero owing to their positive stubbornness $\left(1-\theta_{i}\right)$.

Remark 2. According to Theorem III.2 part (i), the smaller individuals susceptibility $\left(\theta_{i}\right.$ 's), the faster system tends to democracy, that is, equal self-weights. Moreover, since individuals' stubbornness values on one issue have a direct relation with their self-confidence on the next, a group with stubborn individuals never reaches autocracy. Autocracy is defined to be a system where one person has full authority and her decisions are not subject to any external factor.

Lemma III.3 (Existence and uniqueness of equilibria). Consider a cFJ system with $n \geq 2$ such that $\theta_{i}<1$ for all $i \in\{1, \ldots, n\}$. Then, the equilibrium self-weight vector exists and belongs to the interior of $\Delta_{n}$. Moreover, if $\theta_{i} \leq 1 / 3$, then this equilibrium is unique.

Proof. According to Theorem III.2, the dynamics of the cFJ system can be represented by function $F$ which maps any self-weight vector $x \in \Delta_{n}$ to the unique positive dominant left eigenvector of $U(x)$, equation (4). Therefore, if there exists an equilibrium self-weight vector $x^{*}$, where $x^{*}=$ $F\left(x^{*}\right)$, then $x^{*}$ interior $\Delta_{n}$. It is shown in [16] that if the entries of a square matrix $A(t)$ are real analytic functions of parameter $t$ and $\lambda(t)$ is a simple eigenvalue at $t=\tau$ with a corresponding unit eigenvector $\nu(t)$, then for all $t$ near $\tau$ there is a corresponding eigenvalue and unique (normalized) eigenvector whose entries are also real analytic functions of $t$. The entries of matrix $U(x)$ are linear functions of $x$ 's entries, and thus $F(x)$ is a real analytic function of $x$. Furthermore, according to Brouwer fixed-point theorem, any continuous function that maps a convex compact subset of a Euclidean space to itself has a fixed point on that subset. Therefore, $F(x)$ contains at least one equilibrium vector $x \in$ interior $\Delta_{n}$ :

$$
x^{T}\left(I_{n}+\left(I_{n}-\Theta\right)^{-1} \Theta\left(I_{n}-X\right)\left(I_{n}-C\right)\right)=\frac{\mathbb{1}_{n}^{T}}{n} .
$$

Now by contradiction assume there exist two equilibrium vectors $x, z \in$ interior $\Delta_{n}$. Subtracting equation (6) for the two vectors results in

$$
\left(x^{T}-z^{T}\right)\left(I_{n}+\left(I_{n}-X-Z\right)\left(I_{n}-\Theta\right)^{-1} \Theta\left(I_{n}-C\right)\right)=0,
$$

where owing to the commutative property of diagonal matrices and $x^{T} Z=z^{T} X$, we have $x^{T} X-z^{T} Z=\left(x^{T}-z^{T}\right)(X+$ $Z)$. Eigenvalues of $I_{n}-C$ are non-negative and their real parts belong to the interval $[0,2]$. Hence, the eigenvalues of matrix $N:=I_{n}+\left(I_{n}-X-Z\right)\left(I_{n}-\Theta\right)^{-1} \Theta\left(I_{n}-C\right)$ satisfy the following for a negative $1-x_{i}-z_{i}, \operatorname{eig}(N) \geq$ $1+2 \theta_{i}\left(1-x_{i}-z_{i}\right) /\left(1-\theta_{i}\right)$. Since $1-x_{i}-z_{i}>-1$ (note that if $x_{i}=z_{i}=1$, then $\left.x=z=\mathbb{e}_{i}\right)$ and assumption $\theta_{i} \leq 1 / 3$ implies that $\theta_{i} /\left(1-\theta_{i}\right) \leq 1 / 2$, above equation results in $\operatorname{eig}(N)>0$. Therefore, the matrix $N$ is non-singular and it follows from equation (7) that $x^{T}-z^{T}=0$.

Conjecture III.1 (General convergence of cFJ systems). The numerical simulations of $\mathrm{cFJ}$ systems illustrate that for several values of susceptibilities $\left(\theta_{i} \in[0,1)\right)$ and network topologies, all trajectories converge. Moreover, the attractive equilibrium self-weights vector only depends on individuals' susceptibility and relative interpersonal weights.

\section{Doubly-stochastic Relative Interactions}

A doubly-stochastic relative interaction matrix in a group of decision makers indicates a fair system where the total attention that each member receives from all members is uniform and unbiased. For such interaction matrices, [14] established that the DeGroot-Friedkin system (with no stubbornness) converges to the unique final state of equal selfweights, a notion of democracy. In this paper, considering heterogeneous susceptibilities for decision makers can prevent reaching a democracy in general. We prove that, in a special case, if individuals are equally stubborn toward their initial opinions the system converges to a democratic state. Intuitively speaking, if a uniform and unbiased group of decision makers with different initial self-weight start discussing various topics, then they eventually attain identical self-weights.

Lemma IV.1 (Equilibria wtih doubly-stochastic topology). Consider a cFJ system with $n \geq 3$ such that $\theta_{i}=: \theta$ for any $\theta \in(0,1)$ and all $i \in\{1, \ldots, n\}$. Then, the equilibrium self-weight vector is unique and equals $\mathbb{1}_{n} / n$.

Proof. It can be easily verified that $\mathbb{1}_{n}^{T} / n$ satisfies equation (7) by employing $\mathbb{1}_{n}^{T}\left(I_{n}-C\right)=\mathbb{O}_{n}$, which is due to double stochasticity of $C$. Regarding uniqueness of this 
equilibrium, by contradiction assume that $x \in \Delta_{n}$ is an equilibrium vector of the system and unequal to $\mathbb{1}_{n}^{T} / n$. First, assume that $x_{i}<(n-1) / n$ for all $i \in\{1, \ldots, n\}$. Define $y:=x-\mathbb{1}_{n} / n$ and denote $\theta /(1-\theta)$ by $\alpha$. We have the following statement for a doubly-stochastic $C$ :

$$
\begin{aligned}
& y^{T}\left(I_{n}+\alpha\left(I_{n}-X\right)\left(I_{n}-C\right)\right) \\
&=\frac{\mathbb{1}_{n}^{T}}{n}-\frac{\mathbb{1}_{n}^{T}}{n}\left(I_{n}+\alpha\left(I_{n}-X\right)\left(I_{n}-C\right)\right) \\
& \quad=\frac{\alpha}{n} x^{T}\left(I_{n}-C\right)=\frac{\alpha}{n} y^{T}\left(I_{n}-C\right),
\end{aligned}
$$

which is equivalent to $y^{T}=y^{T}\left(\alpha\left((n-1) I_{n} / n-X\right)(C-\right.$ $\left.\left.I_{n}\right)\right)$. As $(n-1) / n-x_{i}>0, \alpha>0$, and all eigenvalues of $\left(C-I_{n}\right)$ have non-positive real parts, the matrix $\alpha((n-$ 1) $\left.I_{n} / n-X\right)\left(C-I_{n}\right)$ can not own an eigenvalue equal to 1. This implies that $y=\mathbb{0}$ and $x=\mathbb{1}_{n} / n$ is the unique equilibrium. Second, if there exists one $x_{i}$ such that $x_{i} \geq$ $(n-1) / n$, then $\sum_{j=1, j \neq i}^{n} x_{j} \leq 1 / n$. Then, equation (6) results in

$$
x_{i}-1 / n=-\alpha\left(x_{i}\left(1-x_{i}\right)-\sum_{j \neq i} c_{j i} x_{j}\left(1-x_{j}\right)\right) .
$$

It is noted that $\sum_{j \neq i} c_{j i}=1$ and since $x_{i} \geq(n-1) / n$, $x_{i}\left(1-x_{i}\right)>\max _{j} x_{j}\left(1-x_{j}\right)$. Therefore, the right hand side of equation (8) is negative, which implies that $x_{i} \leq 1 / n$, which is a contradiction for $n \geq 3$.

Theorem IV.2 (Convergence with doubly-stochastic topology). Consider a cFJ system with $n \geq 3$ such that $\theta_{i}=: \theta$ for any $\theta \in(0,1)$ and all $i \in\{1, \ldots, n\}$. Then, the system exponentially converges to its unique equilibrium self-weight vector $\mathbb{1}_{n} / n$.

Proof. For any $s \in \mathbb{N}$, let us denote $x^{T}(s)-\mathbb{1}_{n}^{T} / n$ by $\Delta(s), \operatorname{diag}(x(s))$ by $X(s)$, and $\theta /(1-\theta)$ by $\alpha$. Then, from equation (5)

$$
\begin{gathered}
\Delta(s+1)=\alpha x^{T}(s+1)\left(I_{n}-X(s)\right)\left(C-I_{n}\right) \\
=\alpha \Delta(s+1)\left(I_{n}-X(s)\right)\left(C-I_{n}\right)+\alpha \frac{\mathbb{1}_{n}^{T}}{n}\left(I_{n}-X(s)\right)\left(C-I_{n}\right) \\
\Rightarrow \Delta(s+1)\left(I_{n}+\alpha\left(I_{n}-C\right)\right) \\
=\alpha \Delta(s)\left(I_{n}-C\right) / n+\alpha \Delta(s+1) \Delta(s)\left(I_{n}-C\right) \\
=\alpha \Delta(s)\left(I_{n} / n+\operatorname{diag}(\Delta(s+1))\left(I_{n}-C\right) .\right.
\end{gathered}
$$

Knowing that $I_{n}-C$ 's eigenvalues are non-negative, $I_{n} / \alpha+$ $I_{n}-C$ is invertible, and $I_{n} / n+\operatorname{diag}(\Delta(s+1))=X(s+1)$, $\Delta(s+1)=\Delta(s) X(s+1)\left(I_{n}-C\right)\left(I_{n} / \alpha+I_{n}-C\right)^{-1}$. Here, we establish that $\|\Delta(s)\|_{2}$ is a strictly decreasing function of $s$. Denoting $1 / \alpha+1$ by $\beta$ we have

$$
\begin{aligned}
A:=\left(I_{n}-C\right)\left(\frac{I_{n}}{\alpha}+I_{n}-C\right)^{-1} & \\
=\left(\beta I_{n}-C\right. & \left.+(1-\beta) I_{n}\right)\left(\beta I_{n}-C\right)^{-1} \\
& =I_{n}+(1-\beta)\left(\beta I_{n}-C\right)^{-1}=: B .
\end{aligned}
$$

Since $\operatorname{eig}(C) \in[-1,1]$, eig $\left(\left(\beta I_{n}-C\right)^{-1}\right) \in\left[\frac{1}{\beta+1}, \frac{1}{\beta}-\right.$ $1]$, and thus eig $B \in[0,2 /(\beta+1)]$. Owing to $\frac{2}{\beta+1}<1$, $\|A\|_{2}<2 /(\beta+1)$, hence, $\|\Delta(s+1)\|_{2}<\|\Delta(s)\|_{2} \| X(s+$
1) $\left\|_{2}<\frac{2}{\beta+1}\right\| \Delta(s) \|_{2}$. Since $\frac{2}{\beta+1}$ is issue independent, this convergence is exponential.

Remark 3. The uniform self-weights vector $\mathbb{1}_{n}^{T} / n$ is an equilibrium vector of a cFJ system if and only if the relative interaction matrix is doubly stochastic.

\section{Centralized Relative Interactions}

A relative interaction network with star topology in a group of decision makers indicates a system where all members trust only a center member besides themselves. This scenario represents centralized social system structuring with rigid hierarchy, which gives rise to inherent mistrust of non-center individuals in their own potentials and loss of self-weight [20]. In a star topology, all nodes assign weight to only one node, the center node (without loss of generality, node 1), and the center node assigns weights $\left(s_{2}, \ldots, s_{n}\right) \in \Delta_{n-1}$ to others. It is established that when individuals are fully susceptible to interpersonal influence $\left(\Theta=I_{n}\right)$, the relative interaction matrix with star topology leads to autocracy, that is, the self-weights vector converges to $\mathbb{e}_{1}$ [14]. In [21], it is claimed that "individuals high in selfweight have been found to believe they are superior to others in many domains, to be self-confident in their viewpoints and actions, and, thus, to be willing to voice their opinion." However, individuals' stubbornness in cFJ systems prevents reaching autocracy, see Figure 1. First, for a cFJ system with heterogeneous stubbornness, we establish that if the center individual is stubborn enough, then the equilibrium selfweights vector is unique and all the non-central individuals obtain self-weights less than half. Next, for a $\mathrm{cFJ}$ system with homogeneous stubbornness, we prove that the center individual obtains the highest self-weight independent of how susceptible she is toward her peers.

Proposition V.1 (Equilibria for star shaped interactions). Consider a cFJ system whose relative interaction matrix has star topology with center node $1, n \geq 3$, and $\theta_{1} \leq 2 / 5$. Then, the equilibrium self-weight vector $x \in$ interior $\Delta_{n}$ exists, is unique, and $x_{i}<1 / 2$ for all $i \in\{2, \cdots, n\}$.

Proof. From Lemma III.3, we know that this system contains at least one equilibrium vector in the interior of $\Delta_{n}$. Here, we prove uniqueness. Since node 1 is the center node, the first row of $C$ matrix is $\left[\begin{array}{llll}0 & s_{2} & \ldots & s_{n}\end{array}\right]$, where $\left(s_{2}, \ldots, s_{n}\right) \in \Delta_{n-1}$, and all other rows are $\left[\begin{array}{llll}1 & 0 & \ldots & 0\end{array}\right]$. Let us denote $\theta_{i} /\left(1-\theta_{i}\right)$ by $\alpha_{i}$. From equation (6), the equilibrium vector $x \in$ interior $\Delta_{n}$ for all $i \in\{2, \ldots, n\}$ satisfies $x_{i}+\alpha_{i} x_{i}\left(1-x_{i}\right)-\alpha_{1} s_{i} x_{1}\left(1-x_{1}\right)=$ $1 / n$. Equivalently, for some $p \in\left(0, \alpha_{1} / 4\right]$,

$$
\alpha_{1} x_{1}\left(1-x_{1}\right)=p \text { and } x_{i}+\alpha_{i} x_{i}\left(1-x_{i}\right)=\frac{1}{n}+s_{i} p .
$$

Hence, $x^{T}\left(\left(I_{n}-X\right) \Theta\left(I_{n}-\Theta\right)^{-1}+\operatorname{diag}([0,1, \cdots, 1])\right)=$ $p\left[1, s_{2}, \cdots, s_{n}\right]+[0,1, \cdots, 1] / n$. Moreover, according to equation (9) for any $i \neq 1, x_{i}<1 / n+s_{i} \alpha_{1} x_{1}\left(1-x_{1}\right) \leq$ $1 / n+\alpha_{1} / 4 \leq 1 / 3+\alpha_{1} / 4$, and since $\theta_{1}<2 / 5, x_{i}<1 / 2$.

Regarding uniqueness of equilibrium vector by contradiction assume that there exist two equilibrium vectors $x, z \in$ 
interior $\Delta_{n}$ that satisfy equation (9), then

$$
\begin{array}{r}
\left(x^{T}-z^{T}\right)\left(\left(I_{n}-X-Z\right) \Theta\left(I_{n}-\Theta\right)^{-1}+\operatorname{diag}([0,1, \cdots, 1])\right) \\
=(p-q)\left[1, s_{2}, \cdots, s_{n}\right],
\end{array}
$$

where $q:=\alpha_{1} z_{1}\left(1-z_{1}\right)$ for some $q \in\left(0, \alpha_{1} / 4\right]$ and without loss of generality $p \geq q$.

First, we show that $p \neq q$ for unequal $x$ and $z$. By contradiction, if $p=q$, then $x_{1}\left(1-x_{1}\right)=z_{1}\left(1-z_{1}\right)$ and for any $i \in\{2, \cdots, n\}, x_{i}\left(1-\theta_{i} x_{i}\right)=z_{i}\left(1-\theta_{i} z_{i}\right)$. For each equation two solutions exists, $x_{1}=z_{1}$ or $x_{1}=1-z_{1}$ and $x_{i}=z_{i}$ or $x_{i}=1 / \theta_{i}-z_{i}$. Since $x$ and $z$ are unequal normalized vectors, there exits at least two nodes $i, j \in$ $\{1, \ldots, n\}$ such that $x_{i} \neq z_{i}$ and $x_{j} \neq z_{j}$. If $x_{1}=1-z_{1}$, then there exists $i \in\{2, \cdots, n\}$ such that $x_{i}=1 / \theta_{i}-z_{i}$. Hence, $x_{1}+x_{i}=1-z_{1}+1 / \theta_{i}-z_{i}>1$, which contradicts $x \in \Delta_{n}$. A similar approach can be employed for any two $i, j \in\{2, \cdots, n\}$.

Second, we establish that $p>q$ contradicts the existence of two non-equal equilibria $x$ and $z$. Note that if $x_{i}+z_{i} \leq 1$ for all $i \in\{1, \ldots, n\}$, then since $(p-q) s_{i} \geq 0, x_{i} \geq z_{i}$ for all $i \in\{1, \ldots, n\}$, which owing to $x, z \in \Delta_{n}$ results in $x=z$, that contradicts the assumption of $p \neq q$. Therefore, there exists at least and at most (since $x, z \in \Delta_{n}$ ) one individual $m$ with $x_{m}+z_{m}>1$ and $x_{m}<z_{m}$. For such $m$, two cases exists: (i) If $m \neq 1$, then $s_{m}>0$ and $x_{m}-z_{m}<0$ requires $\alpha_{m}\left(1-x_{m}-z_{m}\right)+1<0$. However, as proved above, $x_{m}, z_{m}<1 / 2$, and thus $1-x_{m}-z_{m}$ is a positive term, which is a contradiction. (ii) If $i=1$, then equation (6) results in $\left(x_{1}-z_{1}\right)\left(1+\alpha_{1}\left(1-x_{1}-z_{1}\right)\right)=\sum_{2}^{n}\left(x_{i}-z_{i}\right)\left(1+\alpha_{i}(1-\right.$ $\left.x_{i}-z_{i}\right)$ ). As discussed above, for all $i \neq 1, x_{i}+z_{i}<1$ and $x_{i} \geq z_{i}$, hence the right hand side is non-negative. Knowing that $x_{1}<z_{1}$, above equation requires $1+\alpha_{1}\left(1-x_{1}-z_{1}\right) \leq 0$. The assumption of $\theta_{1} \leq 2 / 5<1 / 2$ implies that $\alpha_{1}<1$, and thus $x_{1}+z_{1} \geq 2$, which is a contradiction since $x, z \in$ interior $\Delta_{n}$.

Lemma V.2 (Star shaped with homogenous susceptibilities). Consider a cFJ system whose relative interaction matrix has star topology, $n \geq 3$, and $\theta_{i}=\theta$ for any $\theta \in(0,1)$ and all $i \in\{1, \ldots, n\}$. Then, for any equilibrium self-weight vector, the center node acquires the highest self-weight.

For brevity, we skip the proof to Lemma V.2.

\section{CONCLUSion AND Future Work}

In this paper, we modeled the evolution of the influence network in a group of decision makers via Friedkin's reflected appraisal mechanism and developed a well-posed dynamical system for the evolution of self-weights (social powers). The main challenging future direction for this work is demonstrating our conjecture which leads to the characterization of individuals final self-weights and their dependence on system's parameters.

\section{REFERENCES}

[1] D. Acemoglu, G. Como, F. Fagnani, and A. Ozdaglar. Opinion fluctuations and disagreement in social networks. Mathematics of Operation Research, 38(1):1-27, 2013.

[2] V. D. Blondel, J. M. Hendrickx, and J. N. Tsitsiklis. On Krause's multi-agent consensus model with state-dependent connectivity. IEEE Transactions on Automatic Control, 54(11):2586-2597, 2009.
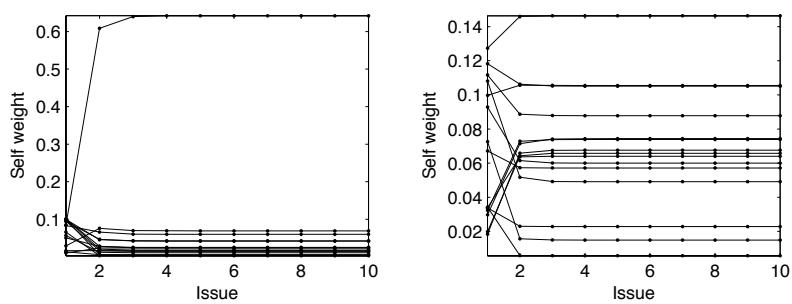

Fig. 1. Illustrates two evolutions of self-weights across issues for $\mathrm{cFJ}$ systems with star shaped interaction networks. In the left plot, the center node has low susceptibility and high stubbornness $\left(\theta_{1}=0.9\right)$ and hence the system tends toward reaching an autocratic state. In the right plot, the center node has low stubbornness $\left(\theta_{1}=0.1\right)$ and this flexibility gives rise to a comparatively democratic state, where the center node's final self-weight is close to the average self-weight in the group despite her central position on the network.

[3] C. H. Cooley. Human Nature and the Social Order. Scribner's, New York, 1902

[4] G. Deffuant, D. Neau, F. Amblard, and G. Weisbuch. Mixing beliefs among interacting agents. Advances in Complex Systems, 3(1/4):8798, 2000.

[5] M. H. DeGroot. Reaching a consensus. Journal of the American Statistical Association, 69(345):118-121, 1974.

[6] P. Frasca, C. Ravazzi, R. Tempo, and H. Ishii. Gossips and prejudices: Ergodic randomized dynamics in social networks, 2013. Available at http://arxiv.org/pdf/1304.2268.pdf.

[7] J. R. P. French. A formal theory of social power. Psychological Review, 63(3):181-194, 1956.

[8] N. E. Friedkin. A formal theory of reflected appraisals in the evolution of power. Administrative Science Quarterly, 56(4):501-529, 2011.

[9] N. E. Friedkin and E. C. Johnsen. Social influence networks and opinion change. In E. J. Lawler and M. W. Macy, editors, Advances in Group Processes, volume 16, pages 1-29. JAI Press, 1999.

[10] F. Gebali. Analysis of Computer and Communication Networks. Springer, 2008.

[11] V. Gecas and M. L. Schwalbe. Beyond the looking-glass self: Social structure and efficacy-based self-esteem. Social Psychology Quarterly, 46(2):77-88, 1983.

[12] F. Harary. A criterion for unanimity in French's theory of social power. In D. Cartwright, editor, Studies in Social Power, pages 168-182. University of Michigan, 1959.

[13] R. Hegselmann and U. Krause. Opinion dynamics and bounded confidence models, analysis, and simulations. Journal of Artificial Societies and Social Simulation, 5(3), 2002.

[14] P. Jia, A. MirTabatabaei, N. E. Friedkin, and F. Bullo. On the dynamics of influence networks via reflected appraisal. In American Control Conference, pages 1251-1256, Washington, DC, USA, June 2013.

[15] P. Jia, A. MirTabatabaei, N. E. Friedkin, and F. Bullo. Opinion dynamics and the evolution of social power in influence networks. March 2013. Submitted.

[16] P. D. Lax. Linear Algebra. Pure and Applied Mathematics. Wiley, 1997.

[17] M. McPherson, L. Smith-Lovin, and J. M. Cook. Birds of a feather: Homophily in social networks. Annual Review of Sociology, 27:415444, 2001.

[18] A. MirTabatabaei and F. Bullo. Opinion dynamics in heterogeneous networks: Convergence conjectures and theorems. SIAM Journal on Control and Optimization, 50(5):2763-2785, 2012.

[19] M. Mobilia, A. Petersen, and S. Redner. On the role of zealotry in the voter model. Journal of Statistical Mechanics: Theory and Experiments, 128:447-483, 2007.

[20] J. L. Pierce and D. G. Gardner. Self-esteem within the work and organizational context: A review of the organization-based self-esteem literature. Journal of Management, 30(5):591-622, 2004.

[21] C. Schoel, M. Bluemke, P. Mueller, and D. Stahlberg. When autocratic leaders become an option-uncertainty and self-esteem predict implicit leadership preferences. Journal of Personality and Social Psychology, 101(3):521-540, 2011.

[22] K.-T. Yeung and J. L. Martin. The looking glass self: An empirical test and elaboration. Social Forces, 81(3):843-879, 2003. 